\title{
Safety and Short-term Efficacy of a Laparoscopic Complete Mesoco- lic Excision for the Surgical Treatment of Right Hemicolon Cancer
}

\author{
Ting Li*, Xiang-Ling Meng, Wei Chen \\ Department of Gastrointestinal Surgery, the First Affiliated Hospital of Anhui Medical University, Hefei, 230022, China.
}

\section{Abstract}

Objective: This study was designed to evaluate the safety and short-term efficacy of a laparoscopic complete mesocolic excision (CME) in patients with right hemicolon cancer.

Methods: A total of 88 patients with right hemicolon cancer were retrospectively reviewed. Forty patients underwent laparoscopic CMEs, and 48 patients underwent open CMEs. The clinical data were analyzed and the outcomes were compared between the two groups.

Result: There was no significant difference between the laparoscopic CME group and open CME group with regard to the harvested lymph node number $(16.60 \pm 3.20$ vs. $15.33 \pm 3.10$, respectively, $\mathrm{P}=0.060)$, hospital stay length $(18.15 \pm 5.17$ days vs. $17.21 \pm 4.47$ days, respectively, $\mathrm{P}=0.360)$, and postoperative complications $(10.0 \%$ vs. $10.4 \%$, respectively, $\mathrm{P}=0.950)$. The operation time in the laparoscopic CME group ( $3.02 \pm 0.55$ hours) was significantly longer than that in the open CME group (2.58 \pm 0.50 hours, $\mathrm{P}=0.004)$. The time to first flatus $(3.28 \pm 0.75$ days vs $3.92 \pm 0.71$ days, respectively, $\mathrm{P}=0.001)$ and getting out of bed time $(1.95 \pm 0.75$ days vs $3.54 \pm 0.71$ days, respectively, $\mathrm{P}=0.001$ ) were both earlier in the laparoscopic CME group than in the open CME group.

Conclusion: A laparoscopic CME is a safe and effective minimally invasive surgery for the treatment of right hemicolon cancer.

Keywords: colon cancer; complete mesocolic excision; laparoscope

\section{INTRODUCTION}

Colon cancer is a common gastrointestinal malignancy, and it is becoming more common among younger age groups ${ }^{[1]}$. There are several therapeutic options for the treatment of colon cancer. However, surgery is the most frequently used and efficient method for all stages of colon cancer ${ }^{[2]}$. The concept of a total mesorectal excision (TME) was first introduced in 1986 by Heald and Ryall, and it was considered to be the standard surgical treatment for rectal cancer ${ }^{[3]}$. In 2009, Hohenberger et al. first proposed that a TME could be translated into a surgical treatment for colon cancer, and this was called a complete mesocolic excision (CME) ${ }^{[4]}$. This technique is performed in order to obtain a complete separation of the mesocolon from the parietal plane, while the supplying arteries and draining veins are ligated at their roots. This technique has been proven to achieve lower local recurrence rates and better overall survival. With the development of the $\mathrm{CME}$, radical resections for co-

\section{Corresponding author: Ting Li}

Mailing address: Department of Gastrointestinal Surgery, the First Affiated Hospital of Anhui Medical University, Hefei 230022, China

Address: Jixi Road No.218, Hefei, Anhui, 230022, China

E-mail: tingli_022@163.com lon cancer have improved over the last decade ${ }^{[5,6]}$. Increasing evidence has reported that CMEs contribute to a decreased recurrence rate and increased five-year survival ${ }^{[4,7,8]}$. At present, a CME is considered to be the standard surgical method for treating colon cancer.

Laparoscopy is a minimally invasive technique that can allow one to visualize the inside of the abdominal cavity with a special camera, and the application of laparoscopic surgery was a significant revolution in clinical practice ${ }^{[9]}$. When compared with traditional open surgery, a laparoscopic surgery has the advantages of a smaller incision, less pain, and a more rapid recovery. Moreover, laparoscopy has been widely used in gastrointestinal malignancy surgeries. A CME can also be guided via a laparoscope, and this procedure is called a laparoscopic CME. It has been reported that a laparoscopic CME provides a better prognosis for colon cancer treatment ${ }^{[10,11]}$. Feng et al. first introduced the laparoscopic CME procedure for right hemicolon cancer [12]; however, the literature regarding this technology for the surgical treatment of right hemicolon cancer is relatively sparse.

In this study, the clinical data of patients with right hemicolon cancer who underwent laparoscopic CMEs was retrospectively reviewed, and the safety and shortterm efficacy were evaluated. 


\section{METHODS}

\section{General patient information}

A total of 88 patients with right hemicolon cancer were enrolled from January 2012 through December 2015. Forty patients underwent laparoscopic CMEs and 48 patients underwent open CMEs. Those cases with no history of chemotherapy and/or radiotherapy underwent enteroscopies, and they were diagnosed with colon cancer via a pathological diagnosis. Those patients with distant metastases and unresectable tumors were excluded via the examination of chest x-ray films, chest computed tomography (CT) scans, abdominal and pelvic brightness (B) scan ultrasonography, CT scans, and/ or magnetic resonance imaging (MRI). The surgery was chosen after full communication with the patients and their dependents. The general information of the patients in both groups is shown in Table 1.

Surgical procedure

Table 1 . General information of patients

\begin{tabular}{clll}
\hline & $\begin{array}{l}\text { Laparosc } \\
\text { opicCME }\end{array}$ & Open CME & Pvalue \\
\hline Cases & 40 & 48 & \\
Age (years) & 59.52 & 60.81 & 0.611 \\
& \pm 12.42 & \pm 11.63 & \\
Gender & & & 0.508 \\
$\quad$ Female & 18 & 25 & \\
Male & 22 & 23 & 0.872 \\
TNM stage & & & \\
I & 4 & 4 & \\
II & 27 & 34 & \\
III & 9 & 10 & \\
\hline
\end{tabular}

\section{Laparoscopic CME}

After administering general anesthesia, the patient was placed in the supine position with their legs apart. A $10-\mathrm{mm}$ trocar was inserted into the umbilical region for the camera port, and a 12-mm trocar was inserted into the left midclavicular line $5 \mathrm{~cm}$ bellow the costal margin for the main operating port. The 5-mm trocars were inserted into the anterior superior iliac spine on both sides and the midpoint of the umbilical cord, as well as the right costal arch midclavicular line $5 \mathrm{~cm}$ below the costal margin for the assistant operating ports. The pneumoperitoneum pressure was maintained at 13 to $14 \mathrm{mmHg}$.

A routine abdominal exploration was performed to confirm the tumor location. After determining the locations of the superior mesenteric vein and ileocolic vessel, the right mesocolon was incised up to Toldt's space, and the duodenum was exposed using an ultrasound knife $2 \mathrm{~cm}$ below the ileocolic vessel. An incision was made in the posterior peritoneum along the front left side of the superior mesenteric vein, and the pericolic mesenteric lymph nodes and adipose tissue were dissected. The ileocolic and right colic artery and vein were fully exposed and skeletonized, and the vessel roots were ligated using a Hem-o-lock vascular clamp. The ascending mesorectum was raised, and the fusion fascial space between the posterior mesorectum (Toldt's fascia) and the prerenal fascia (Gerota's fascia) was entered. An incision was made along the fusion fascial space up to the junction of the ascending colon and the lateral abdominal wall, being careful not to damage to the ureters and reproductive blood vessels. After dissociating upward to expose the head of the pancreas and the descending and horizontal parts of the duodenum, the lymph nodes in front of the pancreas and the surrounding lymph nodes in the right gastroepiploic vessels were removed. The surgical trunk was exposed at the roots of the mesentery, and its surrounding lymph nodes and adipose tissue were removed. The right branches of the blood vessels in the colon were located and cut. If the tumor was located next to the middle of the transverse colon, the roots of the blood vessels in the colon were interrupted using a clip. An ultrasonic blade was used to cut the right omentum majus to the right of the gastric retinal vascular arch, continuing on to incise the hepatocolic ligament and the right ligamentum phrenicocolicum towards the right. An incision was then made downward on the right side of the peritoneum of the ascending colon along the right paracolic sulcus. This incision met the medial separating surface, completing the dissociation of the right colon and its entire mesentery. The specimens from the excision underwent an ilecolostomy, the abdominal cavity was rinsed with distilled water, and a drainage tube was placed under the right side of the liver.

\section{Open CME}

The cecum, ascending colon, flexura hepatica coli, right transverse colon, and mesangial vessels and lymph nodes were removed. Five centimeters of the distal ileum and omentum majus were also removed. The surgical procedure was consistent with the main points of a CME, and it was performed via the sharp incision of the fascia and high ligation of the central vessels. The lymph nodes in the roots of the mesenteric vessels were carefully removed.

\section{Data collection}

The data from all the patients undergoing the CMEs was recorded and compared between the two groups, including the operation time, getting out of bed time, dissected lymph node number, time to first flatus, and postoperative complications. Each patient received a follow-up in order to determine any tumor recurrenc- 
Table 2. Postoperative recovery

\begin{tabular}{llll}
\hline & Laparoscopic CME & Open CME & P value \\
\hline Operation time (h) & $3.02 \pm 0.55$ & $2.58 \pm 0.50$ & 0.004 \\
Harvested lymph node number & $16.60 \pm 3.20$ & $15.33 \pm 3.10$ & 0.060 \\
Length of hospital stay (d) & $18.15 \pm 5.17$ & $17.21 \pm 4.47$ & 0.360 \\
Getting out-of-bed time(d) & $1.95 \pm 0.75$ & $3.54 \pm 0.71$ & $<0.001$ \\
Time to first flatus(d) & $3.28 \pm 0.75$ & $3.92 \pm 0.71$ & $<0.001$ \\
\hline
\end{tabular}

es or metastases. The duration of the follow-up ranged from 1 to 46 months.

\section{Statistical analysis}

The statistical analysis was performed using SASS 12.0 software. The categorical data were compared using a $\chi 2$ test, and the measurement data were compared with an independent samples t test. A P value of less than 0.05 was considered to be statistically significant.

\section{RESULTS}

\section{Surgical procedure}

The general information from Table 1 shows that there were no statistical differences in the age, gender, and Tumor, Node, Metastasis (TNM) stage between the laparoscopic CME and open CME groups ( $\mathrm{P}>0.05)$. All of the laparoscopic and open CME operations were finished smoothly, and no surgery-related or perioperative deaths occurred in any of the cases. As shown in Table 2 , the operation time in the open CME group was statistically shorter than that in the laparoscopic CME group ( $3.02 \pm 0.55$ vs. $2.58 \pm 0.50$ hours, $\mathrm{P}=0.004)$. The pathology reports showed no statistical difference in the harvested lymph node number between the two groups (16.60 \pm 3.20 vs. $15.33 \pm 3.10$, respectively, $\mathrm{P}=0.060$ ).

Table 2 Postoperative recovery

\section{Postoperative recovery}

The length of the hospital stay for the patients in the laparoscopic CME group was similar to that in the open CME group (18.15 \pm 5.17 vs. $17.21 \pm 4.47$ days, respectively, $\mathrm{P}=0.360$ ). However, the laparoscopic CME group had a significantly shorter getting out of bed time ( $1.95 \pm 0.75$ vs. $3.54 \pm 0.71$ days, respectively, $\mathrm{P}<0.001)$ and a significantly shorter time to first flatus $(3.28 \pm 0.75$ vs. $3.92 \pm 0.71$ days, respectively, $\mathrm{P}<0.001$ ).

\section{Postoperative complications}

The postoperative complication rate in the laparoscopic CME group was $10.0 \%$ (4/40), and the complications included incision infections $(n=3)$ and an intestinal obstruction $(\mathrm{n}=1)$. The complication rate in the open CME group was $10.4 \%$ (5/48), including incision infections $(n=3)$, a pulmonary infection $(n=1)$, and postoperative anastomotic bleeding $(n=1)$. There were no anastomotic fistulas in either group. Moreover, there was no statistical difference in the postoperative complication rate between the two groups. During the follow-up (range from 1 to 46 months), there were two tumor recurrence cases in the laparoscopic CME group and three tumor recurrence cases in the open CME group. In addition, one patient in the laparoscopic CME group died, and two patients in the open CME group died during the follow-up. There were no statistical differences in the recurrence rates $(\chi 2=0.06, P=0.80)$ and death rates $(\chi 2=0.18, \mathrm{P}=0.67)$ between the two groups.

\section{DISCUSSION}

The postoperative rectal cancer recurrence rate has been significantly reduced, and the postoperative survival rate has been significantly improved since Heald and Ryall presented the concept of a TME ${ }^{[3,13]}$. In 2009, Hohenberger et al. proposed the standardized application of the CME in the radical resection of colon cancer. They also analyzed a total of 1,329 patients with colon cancer that had undergone CMEs, and they concluded that a CME could significantly reduce the recurrence rate and improve the survival rate ${ }^{[4]}$. Based on the experience of Hohenberger et al., a CME should be performed in the radical resection of colon cancer in order to remove all of the tumors, based on the excision of the complete mesocolon, and achieve a maximum lymph node harvest. The CME has brought the new concept of fine anatomy throughout the entire colon cancer surgery process. We conducted CMEs based on laparoscopic right hemicolon cancer resections, and we observed their short-term efficacy and safety.

After reviewing the clinical data, we discovered that the incision pain was slighter, and the time to get out of bed and time to first flatus was earlier in those patients who underwent laparoscopic CMEs than in those who underwent open CMEs. This may have been due to the minimally invasive laparoscopic surgery, with its small abdominal incision and a slight traumatic reaction, which are beneficial for the rapid recovery of postoperative intestinal function. Its minimally invasive feature is also good for preventing pulmonary complications. In this study, the postoperative complications included incision infections in three cases and an intestinal obstruction in one case, but there were no pulmonary 
infections in any of the patients who underwent the laparoscopic CMEs. The postoperative complication rate showed no statistical difference between the laparoscopic CME and open CME groups. The number of lymph nodes harvested in the laparoscopic CME group was slightly higher than that in the open CME group, but there was no statistical difference, which is in line with other reports ${ }^{[14]}$.

All of the patients received follow-ups in this study. During the follow-up (range from 1 to 46 months), there were two patients with tumor recurrences in the laparoscopic CME group, one of which died. The recurrence rate and death rate in the laparoscopic CME group exhibited no differences when compared with the open CME group. Overall, a laparoscopic CME for the treatment of radical right colon cancer is a minimally invasive surgery that can improve the quality of life of the patient. Therefore, it is concluded that a laparoscopic CME for the treatment of radical right colon cancer is safe and feasible.

In recent years, with the rapid development of laparoscopic techniques, minimally invasive surgery has become increasingly popular. Laparoscopic colorectal cancer surgery, which has an obvious minimally invasive advantage in the abdominal cavity, has become one of the most established surgical methods used for gastrointestinal cancer surgery ${ }^{[15,16]}$. For the better application of CMEs in laparoscopic hemicolectomies for right colon carcinoma cases, the following points have been summarized in the literature ${ }^{[8,12,17,18]}$ and in our clinical experience. First, a CME presents the concept of fine anatomy. A sharp dissection was made along the fusion fascial space between Toldt's fascia and Gerota's fascia, along the pancreaticoduodenal fascia. The gastrocolic ligament was removed under or above the vessel arches of greater curvature, and the right hemicolon and its mesentery were totally excised, which emphasizes the complete resection of the lymph nodes and mesentery ${ }^{[4]}$. Second, the peritoneal tissues of the superior mesenteric vein and arterial surface were opened, and the blood vessels were ligated and cut off in the central area for the most thorough removal of the lymph nodes. It is also beneficial to block the hematogenous spreading pathway of the tumor. The operation does not touch the tumor, and it significantly reduces the extrusion of the tumor, which is more consistent with the tumor-free principle in tumor surgery. Third, the local magnification of the surgical field under laparoscopy is fully utilized, making it more accurate in the surgical approach and at the anatomical level. The enlargement of the laparoscope enables the smaller lymph nodes to be easily identified and removed. Fourth, the use of an ultrasound knife can significantly reduce blood loss, facilitating the dissection at the correct anatomical level and improving the operation. CME operations in laparoscopic hemicolectomies for right colon carcinoma cases have been carried out in the clinic ${ }^{[19,20]}$. They not only elevate the survival rate, but they also improve the quality of life of the patients ${ }^{[10]}$. This procedure has the advantage of being minimally invasive, and the patients can achieve rapid recovery after the surgery. A laparoscopic CME for the treatment of radical right colon cancer has been proven to be safe and feasible, and its short-term curative effect is satisfactory. It is a minimally invasive surgical method with application prospects; however, the long-term efficacy requires further study.

\section{REFERENCES}

1. Siegel, R. L., Miller, K. D., and Jemal, A. (2018) Cancer statistics, 2018. CA: a cancer journal for clinicians 68, 7-30

2. Hasegawa, H., Ueda, S., Nakanoko, T., Gion, T., and Kitamura, M. (2016) [An Examination of Colon Cancer Treatment Policies for the Elderly]. Gan to kagaku ryoho. Cancer \& chemotherapy 43, 1523-1525

3. Heald, R. J., and Ryall, R. D. (1986) Recurrence and survival after total mesorectal excision for rectal cancer. Lancet 1, 1479-1482

4. Hohenberger, W., Weber, K., Matzel, K., Papadopoulos, T., and Merkel, S. (2009) Standardized surgery for colonic cancer: complete mesocolic excision and central ligation--technical notes and outcome. Colorectal disease : the official journal of the Association of Coloproctology of Great Britain and Ireland 11, 354-364; discussion 364355

5. Ozben, V., Baca, B., Atasoy, D., Bayraktar, O., Aghayeva, A., Cengiz, T. B., Erguner, I., Karahasanoglu, T., and Hamzaoglu, I. (2016) Robotic complete mesocolic excision for right-sided colon cancer. Surgical endoscopy 30, 46244625

6. Benz, S., Tam, Y., Tannapfel, A., and Stricker, I. (2016) The uncinate process first approach: a novel technique for laparoscopic right hemicolectomy with complete mesocolic excision. Surgical endoscopy 30, 1930-1937

7. Du, S., Zhang, B., Liu, Y., Han, P., Song, C., Hu, F., Xia, T., Wu, X., and Cui, B. (2018) A novel and safe approach: middle cranial approach for laparoscopic right hemicolon cancer surgery with complete mesocolic excision. Surgical endoscopy 32, 2567-2574

8. Zurleni, T., Cassiano, A., Gjoni, E., Ballabio, A., Serio, G., Marzoli, L., and Zurleni, F. (2018) Surgical and oncological outcomes after complete mesocolic excision in right-sided colon cancer compared with conventional surgery: a retrospective, single-institution study. International journal of colorectal disease 33, 1-8

9. Agrusa, A., Di Buono, G., Buscemi, S., Cucinella, G., Romano, G., and Gulotta, G. (2018) 3D laparoscopic surgery: a prospective clinical trial. Oncotarget 9, 17325-17333 
10. Wang, Y., Zhang, C., Zhang, D., Fu, Z., and Sun, Y. (2017) Clinical outcome of laparoscopic complete mesocolic excision in the treatment of right colon cancer. World journal of surgical oncology 15, 174

11. Mori, S., Baba, K., Yanagi, M., Kita, Y., Yanagita, S., Uchikado, Y., Arigami, T., Uenosono, Y., Okumura, H., Nakajo, A., Maemuras, K., Ishigami, S., and Natsugoe, S. (2015) Laparoscopic complete mesocolic excision with radical lymph node dissection along the surgical trunk for right colon cancer. Surgical endoscopy 29, 34-40

12. Feng, B., Sun, J., Ling, T. L., Lu, A. G., Wang, M. L., Chen, X. Y., Ma, J. J., Li, J. W., Zang, L., Han, D. P., and Zheng, M. H. (2012) Laparoscopic complete mesocolic excision (CME) with medial access for right-hemi colon cancer: feasibility and technical strategies. Surgical endoscopy 26, 36693675

13. Yamaguchi, T., Kinugasa, Y., Shiomi, A., Kagawa, H., Yamakawa, Y., Furutani, A., Manabe, S., Yamaoka, Y., and Hino, H. (2018) Oncological outcomes of robotic-assisted laparoscopic versus open lateral lymph node dissection for locally advanced low rectal cancer. Surgical endoscopy

14. Huang, J. L., Wei, H. B., Fang, J. F., Zheng, Z. H., Chen, T. F., Wei, B., Huang, Y., and Liu, J. P. (2015) Comparison of laparoscopic versus open complete mesocolic excision for right colon cancer. International journal of surgery 23, $12-17$

15. Lainas, P., Dammaro, C., Gaillard, M., Donatelli, G., Tranchart, H., and Dagher, I. (2018) Safety and short-term outcomes of laparoscopic sleeve gastrectomy for pa- tients over 65 years old with severe obesity. Surgery for obesity and related diseases : official journal of the American Society for Bariatric Surgery

16. Liu, X. Z., Yin, K., Fan, J., Shen, X. J., Xu, M. J., Wang, W. H., Zhang, Y. G., Zheng, C. Z., and Zou da, J. (2015) Long-Term outcomes and experience of laparoscopic adjustable gastric banding: one center's results in China. Surgery for obesity and related diseases : official journal of the American Society for Bariatric Surgery 11, 855-859

17. Bernhoff, R., Sjovall, A., Buchli, C., Granath, F., Holm, T., and Martling, A. (2018) Complete mesocolic excision in right-sided colon cancer does not increase severe shortterm postoperative adverse events. Colorectal disease : the official journal of the Association of Coloproctology of Great Britain and Ireland 20, 383-389

18. Yang, X., Wu, Q., Jin, C., He, W., Wang, M., Yang, T., Wei, M., Deng, X., Meng, W., and Wang, Z. (2017) A novel hand-assisted laparoscopic versus conventional laparoscopic right hemicolectomy for right colon cancer: study protocol for a randomized controlled trial. Trials 18, 355

19. Su, C., Hong, X., and Qiu, X. (2017) Laparoscopy-assisted complete mesocolic excision for right-hemi colon cancer. Journal of visualized surgery 3, 28

20. Matsuda, T., Sumi, Y., Yamashita, K., Hasegawa, H., Yamamoto, M., Matsuda, Y., Kanaji, S., Oshikiri, T., Nakamura, T., Suzuki, S., and Kakeji, Y. (2017) Anatomy of the Transverse Mesocolon Based on Embryology for Laparoscopic Complete Mesocolic Excision of Right-Sided Colon Cancer. Annals of surgical oncology 24, 3673 